\title{
EVALUACIÓN DE LOS FACTORES DE RIESGO DE EMBARAZO EN ADOLESCENTES EN LA COMUNIDAD DE LAS TABLAS MUNICIPIO MATANZAS, PROVINCIA PERAVIA, REPÚBLICA DOMINICANA, DURANTE EL PERÍODO NOVIEMBRE 2017 - ENERO 2018 \\ Evaluation of the Risk Factors of Pregnancy in Adolescents in the Community of Las Tablas, Matanzas Municipality, Peravia Province, Dominican Republic, during the peri- od November 2017 - January 2018
}

\section{Amelia Navarro Ramírez*, Bellelyn Domínguez *, Rodríguez. Charlotte Montes de Oca*, Ana Ramírez Díaz*, Nicole Barreto Rojas*, César López**, Emilton López ${ }^{* * *}$}

Fecha de recibido: 12 septiembre, 2018 • Fecha de aprobado: 26 noviembre, 2018

Cómo citar: Navarro Ramírez A, Domínguez B, Rodríguez Charlotte M de O, Ramírez Díaz A, Barreto Rojas N, López C, López E. Evaluación de los Factores de Riesgo de Embarazo en Adolescentes en la Comunidad de Las Tablas. Municipio Matanzas, Provincia Peravia, República Dominicana, durante el período noviembre 2017 - enero 2018. cysa [Internet]. 10 abr. 2019 [citado 12 abr. 2019];1(1):43-0. Available from: https://revistas.intec.edu.do/index.php/cisa/article/view/1330

\section{Resumen}

Introducción: La adolescencia se define como el período de crecimiento y desarrollo humano entre los $10 \mathrm{y}$ 19 ańos. El embarazo precoz es aquel que se produce en este grupo etario, sin importar la madurez o dependencia de su núcleo familiar de origen. En República Dominicana, el 22\% de las adolescentes han estado embarazadas.

Objetivo: Evaluar los factores de riesgo de embarazo en adolescentes en la comunidad de Las Tablas, municipio Matanzas, provincia Peravia, República Dominicana, durante el período noviembre 2017 - enero 2018.

Metodología: Estudio prospectivo, descriptivo, de corte transversal en el que se entrevistaron 45 adolescentes fértiles de la comunidad de Las Tablas.

Resultados: Se obtuvo que un 20\% (29 casos) presentó disfunción familiar como riesgo para desencadenar un embarazo adolescente. De las jóvenes con vida sexual activa, un 100\% (12 casos) indicaron haberla iniciado a los 14 años o más. Un 69\% (31 casos) han recibido información sobre el uso de los métodos anticonceptivos. Cabe destacar que 15 adolescentes (33\%) han sido víctima de violencia en la comunidad.

\footnotetext{
*Médicos Internos, Hospital de Matanzas, República Dominicana. **Ginecólogo-Obstetra. cesar.lopez@intec.edu.do

*** Pediatra. cesar.lopez@intec.edu.do
}

Conclusiones: El embarazo adolescente es una problemática multifactorial, donde predominan la disfunción familiar, ser hija de madre con historia de embarazo adolescente, uso de alcohol y otras sustancias, entre otras.

Palabras clave: Adolescencia; embarazo; anticonceptivos; sexualidad; reproducción.

\section{Abstract}

Adolescence is defined as the period of human growth and development between the ages 10 and 19. An early pregnancy is the one that occurs in this age group, regardless of the maturity or dependence of their family. In the Dominican Republic, 22\% of adolescents have been pregnant. The objective is to assess the risk factors of pregnancy in adolescents in the community of Las Tablas, municipality of Matanzas, province of Peravia, Dominican Republic, during the period from November 2017 to January 2018. This was a prospective, descriptive, cross-sectional study in which 45 fertile adolescents from the Las Tablas community were interviewed.

Amelia.navarro.ramirez@hotmail.com

Bellelynd@gmail.com

Charlottepmdo@gmail.com

Anaramirezd04@gmail.com

Nikkibarreto@hotmail.com emiltonlopez@hotmail.com 
As a result, it was found that $20 \%$ (29 cases) presented family dysfunction as a risk to trigger a teenage pregnancy. Of the girls with active sexual life, 100\% (12 cases) indicated that they started at age 14 or older. $69 \%$ (31 cases) have received information on the use of contraceptive methods. It should be noted that 15 adolescents (33\%) have been victims of violence in the community. In conclusion, adolescent pregnancy is a multifactorial problem, where family dysfunction predominates, along with being the daughter of a mother with history of teenage pregnancy, use of alcohol, among others.

Key words: Adolescence; Pregnancy; Contraceptives; Sexuality; Reproduction.

\section{Introducción}

La Organización Mundial de la Salud (OMS) define la adolescencia como el período de crecimiento y desarrollo humano que se produce después de la niñez y antes de la edad adulta, entre los 10 y los 19 años. Es una de las etapas de transición más importantes, que se caracteriza por un ritmo acelerado de crecimiento y de cambios, que viene condicionada por diversos procesos biológicos, donde se da lugar la maduración física, cognoscitiva y psicosocial. El comienzo de la pubertad marca el pasaje de la niñez a la adolescencia ${ }^{1}$.

La adolescencia se divide en tres etapas: la adolescencia temprana, la media y la tardía. La duración de cada etapa depende de cada individuo y de su cultura, aunque cada una tiene una duración de 3 años aproximadamente que comprende desde los 10 a 13 , desde los 14 a 16 y desde los 17 a 19 , respectivamente ${ }^{2}$.

\section{Embarazo en adolescentes}

El embarazo en la adolescencia es aquel que se produce entre los 10 a los 19 años de edad, independientemente del grado de madurez biológica o psicológica y/o dependencia del adolescente con su núcleo familiar de origen ${ }^{3}$.
En República Dominicana, según el Informe Nacional de Desarrollo Humano (INDH) 2017, el $22 \%$ de las adolescentes han estado embarazadas. Esta tasa es de 34\% más alta que el promedio de los países de América Latina y el Caribe ${ }^{4}$.

Factores de riesgo

El embarazo adolescente es una problemática donde intervienen diferentes factores, entre estos se destacan: biológicos, psciológicos y sociales, del desarrollo cognitivo, entre otros.

\section{Factores biológicos}

Cada vez es más frecuente que los adolescentes entren en la pubertad a menor edad, lo cual incrementa la libido y el interés sexual, incrementando el riesgo de embarazo (2C). Estos desarrollan una sensación de inestabilidad y a veces adoptan actitudes promiscuas, por la enaltecida necesidad del adolescente de explorar su cuerpo y tener experiencias compartidas en este terreno 5 .

\section{Factores psicosociales}

Un mal funcionamiento familiar puede predisponer a una relación sexual prematura. La baja autoestima de una adolescente que sufre de discriminación afectiva, la lleva a buscar afecto a través de las relaciones sexuales, además de que encuentra alivio en el embarazo, ya que le permite salir del hogar en el cual se ve amenazada de violencia. Existen otros riesgos asociados a la familia tales como: la inestabilidad familiar, madre con historia de embarazo adolescente, figuras masculinas cambiantes y enfermedad crónica de uno de los padres ${ }^{6}$.

Es importante señalar la influencia que tienen los medios de comunicación sobre la población adolescente. Estos ejercen una gran presión debido a la inmensa cantidad de estímulos sexuales, así como una pseudo valoración de este como placer, es decir, sin compromiso afectivo ${ }^{7}$. 
El comportamiento del embarazo adolescente remite a un escenario de desigualdades donde las menos educadas, más pobres y residentes en áreas rurales están en desventaja y resultan ser las más afectadas ${ }^{8}$.

El machismo y la necesidad de demostrar masculinidad también llevan a menudo a comportamientos que aumentan los embarazos en este grupo etario, sobre todo en escenarios donde la adolescente se ve en la obligación de satisfacer una pareja que es mayor que ella en edad'.

El hacinamiento, la migración, el estrés, la delincuencia, la exposición a bebidas alcohólicas u otras sustancias, la falta de recursos y acceso a los sistemas de atención en salud, la pérdida de la religiosidad son otros factores que afectan las barreras para el ejercicio de la sexualidad adolescente ${ }^{10}$.

De manera general, a menor educación, mayor proporción de embarazo. Por tanto, la falta de educación sexual en la familia y las escuelas, figura como un factor causal del problema. Esta deficiencia es debido a que existen sanciones sociales y culturales, basadas en mitos y tabúes alrededor del abordaje de la sexualidad ${ }^{11}$.

\section{Consecuencias del embarazo en la adolescente}

A corto plazo, la adolescente se encuentra en riesgo de presentar: anemia, parto prematuro, estado hipertensivo del embarazo, infecciones de transmisión sexual, mortalidad materna. A largo plazo algunas de las consecuencias son: cese del crecimiento, obesidad e hipertensión arterial.

El estado psicológico y social de la madre adolescente también puede verse afectado, aumentando la recidiva de embarazos no deseados, inestabilidad familiar, fracaso escolar y/o bajo nivel educativo y descenso de los ingresos económicos.

\section{Prevención del embarazo en la adolescencia}

Como estrategia se deben tener en cuenta los factores protectores y los factores de riesgo biológicos y psicosociales para implementar una intervención adecuada y oportuna.

En la prevención primaria se debería brindar información sobre fisiología reproductiva y métodos de anticoncepción tanto en las escuelas como en el entorno familiar, hacer una utilización adecuada de los medios de comunicación, fomentar la educación entre los padres y los hijos, brindar entrenamiento a personas que tienen contacto prolongado con adolescentes para la atención de aquellos que presenten situaciones de alto riesgo ${ }^{12}$.

El enfrentamiento de la problemática no puede basarse simplemente en los esfuerzos médicos, más bien requiere de estrategias articuladas y negociadas entre los diferentes sectores, la comunidad y sus líderes, y muy especialmente de los adolescentes ${ }^{13,14}$.

En 2011, la OMS publicó junto con el Fondo de Población de las Naciones Unidas (UNFPA) unas directrices sobre la prevención de los embarazos precoces y la reducción de los resultados negativos para la reproducción. Entre sus objetivos se encontraban: reducir el número de matrimonios en menores de 18 años, aumentar el uso de anticonceptivos en adolescentes sexualmente acitvas, reducir las relaciones forzadas, fomentar la comprensión y apoyo hacia las jóvenes ${ }^{15}$.

\section{Materiales y Métodos}

Este estudio será de tipo prospectivo, realizado durante el período de noviembre 2017- enero 2018, de fuentes primarias; descriptivas, ya que los datos serán detallados tal cual se presenten sin ninguna intervención por parte de los investigadores; de corte transversal, porque las variables se estudiarán en una sola ocasión, sin ofrecerle continuidad una vez acabada la recolección de datos.

La herramienta de recolección de datos será un formulario creado por los autores, con el cual se obtendrá la información requerida de las entrevistas 
a adolescentes de 10 a 19 años residentes en Las Tablas que accedieron a llenar el cuestionario ya mencionado. El mismo incluía las variables de edad, escolaridad, dinámica familiar, dependencia económica, edad de menarquia y de primera relación sexual, uso de sustancias de recreación, educación sobre salud sexual, entre otros.

El universo de esta investigación estuvo constituido por todas las mujeres en edad fértil del municipio Matanzas, provincia Peravia, República Dominicana. La población estuvo conformada por todas las mujeres fértiles que residen en la comunidad de Las Tablas, municipio Matanzas, provincia Peravia, República Dominicana, durante el período noviembre 2017 - enero 2018, representando una totalidad de 344 pacientes. La muestra fueron todas las adolescentes fértiles que residen en la comunidad de Las Tablas, municipio Matanzas, provincia Peravia, República Dominicana, durante el período noviembre 2017 - enero 2018, correspondiente a 45 pacientes.

Para que una paciente pudiese formar parte de la investigación se tomaron en cuenta los siguientes criterios: pacientes entre 10 y 19 años, fértil, residente en la comunidad de Las Tablas y que tuviera una ficha de registro familiar. Se excluyeron un total de 55 pacientes, por tener edad menor a 10 ańos o mayor de 19 ańos, no fértiles, residentes fuera de la comunidad de Las Tablas, y que no tuvieran una ficha de registro familiar completa.

Antes de la aplicación del cuestionario se procedió al consentimiento informado con las pacientes y se aseguró a las encuestadas la absoluta confidencialidad de la información. Los datos obtenidos fueron analizados estadísticamente mediante el programa Microsoft Excel versión Office 2017.

\section{Resultados}

\section{Gráfica No. 1: Factores de riesgo $(\mathrm{N}=45)$}

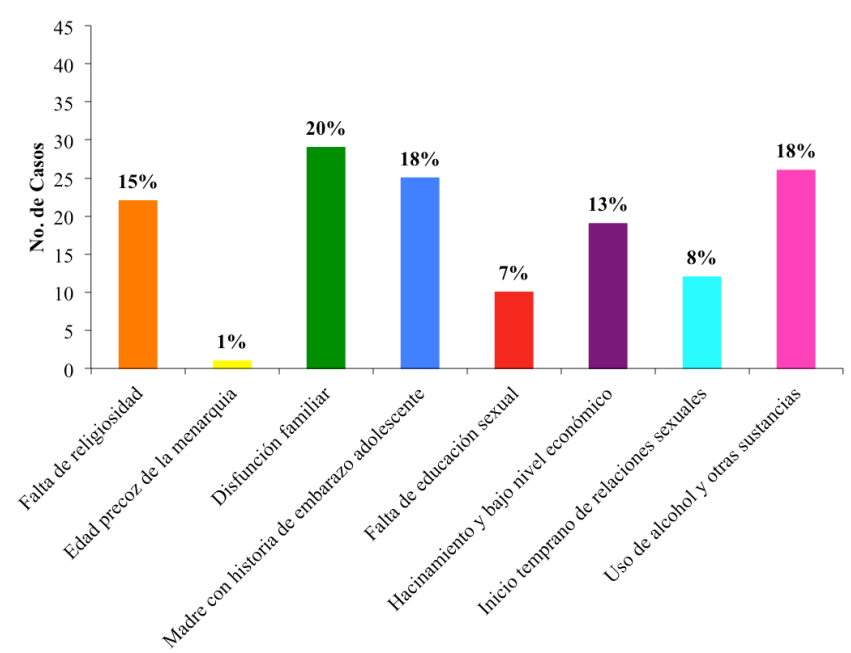

Fuente: Navarro A, Domínguez B, Pilier C, Ramírez A, Barreto N, Dr López C., enero 2018.

Esta gráfica muestra los factores de riesgo de embarazo que presentaron las adolescentes de la comunidad de Las Tablas. En la misma se observa que la disfunción familiar es el factor más común con un $20 \%$ (29 adolescentes). Le siguen en orden de frecuencia el uso de alcohol y otras sustancias y el ser hijas de madre con historia de embarazo adolescente, ambos con 26 casos, siendo esto un $18 \%$. Un $15 \%$ (22 jóvenes) presentaron el factor de riesgo asociado a la falta de religiosidad. 19 adolescentes viven en condiciones de hacinamiento por el bajo nivel socioeconómico (13\%). El inicio temprano de las relaciones sexuales y la falta de educación sexual representaron $8 \%$ (12 casos) y 7\% (10 casos) respectivamente. Solo 1 paciente (1\%) presentó edad precoz de la menarquia. 
Gráfica No. 2: Edad de inicio en las relaciones sexuales $(n=12)$

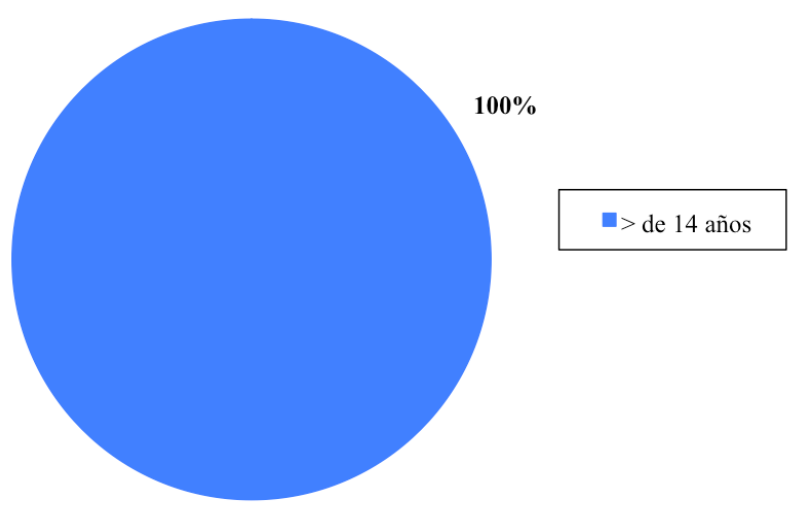

Fuente: Navarro A, Domínguez B, Pilier C, Ramírez A, Barreto N, Dr López C., enero 2018.

De acuerdo con los resultados obtenidos se observa que 12 adolescentes de la muestra ya habían iniciado su vida sexual al momento de aplicada la encuesta. Aunque existían otras opciones para especificar tales como $<10$ años y de 11 a 13 años, el $100 \%$ de las jóvenes indicaron haber tenido su primera relación sexual a los 14 años o más.

\section{Gráfica No. 3: Conocimiento sobre el uso de mé- todos anticonceptivos $(\mathrm{N}=45)$}

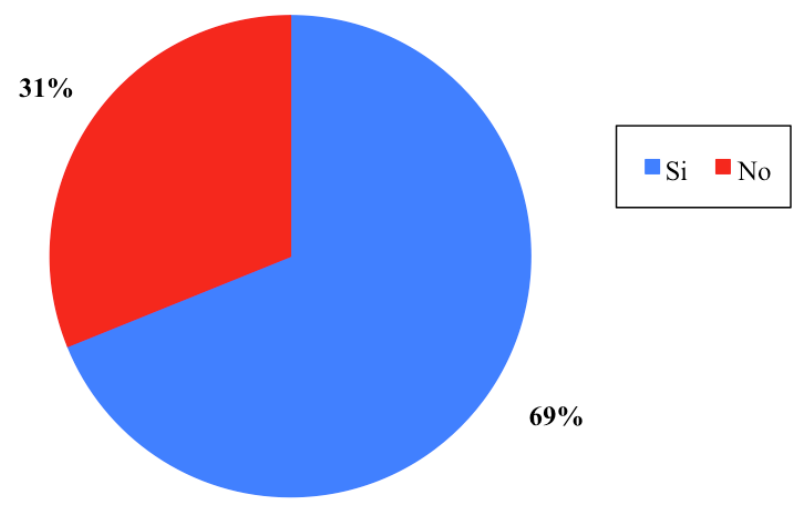

Fuente: Navarro A, Domínguez B, Pilier C, Ramírez A, Barreto N, Dr López C., enero 2018.

La siguiente gráfica hace referencia al conocimiento de las adolescentes sobre el uso de métodos anticonceptivos. La misma se divide en dos grupos. Un primer cohorte correspondiente a aquellas pacientes que no poseen conocimientos suficientes sobre el tema, representado por 14 casos para un $31 \%$. Un 69\%, es decir, 31 adolescentes han recibido información sobre el tema.

\section{Gráfica No. 4: Incidencia de violencia $(\mathrm{N}=45)$}

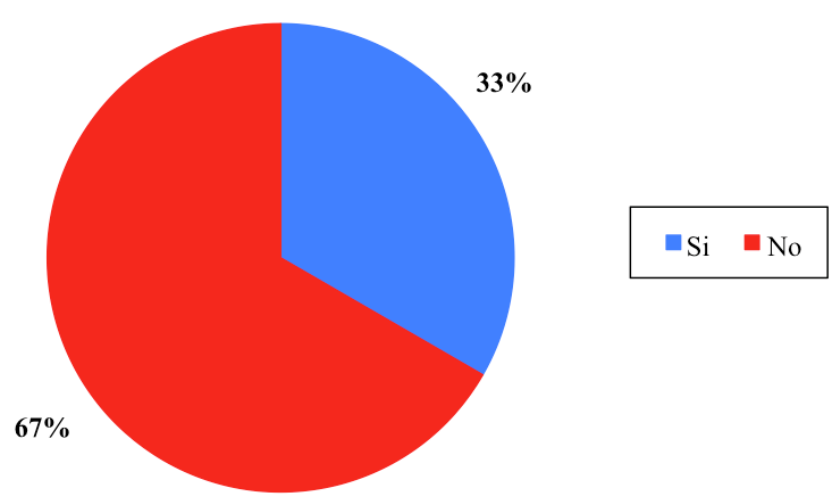

Fuente: Navarro A, Domínguez B, Pilier C, Ramírez A, Barreto N, Dr López C., enero 2018.

Esta gráfica muestra la incidencia de violencia hacia las adolescentes de Las Tablas. En la misma se muestra que un $33 \%$ de las jóvenes ha sido víctima de algún tipo de maltrato, ya sea verbal, físico o sexual, con un total de 15 casos. 30 adolescentes (67\%) indicaron nunca haber sufrido ningún tipo de agresión hacia ellas.

\section{Discusión y Conclusiones}

Las características en las que suelen vivir las adolescentes entrevistadas, es las de jóvenes que viven en un entorno familiar numeroso y desestructurado, donde la figura del padre suele ser inexistente, y la madre tiene antecedente de embarazo adolescente. Suelen ser familias de muy bajos recursos y encontrarse en condiciones de hacinamiento. La educación sexual suele ser muy escasa, tanto en el hogar como en la escuela, ya que piensan que mientras menos aborden el tema de sexualidad, más tarde empezarán sus inquietudes por experimentarla, lo cual es totalmente erróneo, ya que crea un ambiente de confusión e ignorancia en la adolescente. 


\section{Amelia Navarro Ramírez, Bellelyn Domínguez, Rodríguez Charlotte Montes de Oca,}

Ana Ramírez Díaz, Nicole Barreto Rojas, César López y Emilton López

La investigación realizada en el año 2007 en España por el Dr. Gustavo Ávila, "Factores de riesgo asociados al embarazo en adolescentes en comunidades urbanas", asegura tal como lo planteado, que el embarazo en la adolescencia está influenciado por varios factores, aunque el principal sea la falta de educación sobre salud sexual y reproductiva. Esto hace que las prácticas sexuales que experimentan estas adolescentes se den bajo conceptos falsos o verdades a medias que comparten entre los mismos jóvenes ${ }^{16}$.

El factor de riesgo más común de embarazo en adolescentes en este estudio fue la disfunción familiar en un $20 \%$ con 29 casos, lo que concuerda con los datos recopilados por el estudio del Centro Nacional de Investigaciones en Salud Materna Infantil en colaboración con Profamilia en el año 2011, donde se afirma que las adolescentes más afectadas por el embarazo precoz son aquellas provenientes de familias lideradas por mujeres como familias monoparentales, o donde la figura masculina es cambiante ${ }^{13}$.

En las adolescentes sexualmente activas, la edad más frecuente de inicio de relaciones sexuales fue 14 años o más. Esto concuerda con los datos recopilados por Rodríguez, Cala, Nápoles, Milám y Aguilar en el 2017 en el estudio "Factores de riesgo asociados al embarazo en adolescentes" para la revista científica de la Universidad de Guantánamo, donde se observó que las jóvenes poseían 2.3 veces más riesgo de quedar embarazadas si tenían su primera relación sexual entre los 15 y 19 años $^{12}$.

Es más frecuente que las adolescentes tengan un grado de información sobre el uso de los distintos métodos anticonceptivos más comunes, aunque un $31 \%$ con 14 casos indicó no tener ningún tipo de conocimiento sobre el tema. La información recopilada se puede corroborar con los datos proporcionados en el estudio "Conocimiento y uso de métodos anticonceptivos en un grupo de adolescentes" realizado en el año 2014 en España por el Dr. William Casadiego, donde se demostró que alrededor del 38\% de los adolescentes no tienen suficiente información sobre los métodos anticonceptivos $^{13}$.

Una tercera parte de las jóvenes han experimentado algún tipo de violencia. Cabe destacar que se ha demostrado, que existe un rechazo hacia las creencias sobre los actos violentos de género y también desconocimiento sobre cuáles son los tipos de violencia que existen y que puede afectar a las adolescentes. El estudio "Violencia de género en adolescentes" del año 2015, realizado en España por el Dr. Francisco Guzmán, asegura que más del 90\% de las chicas ignoran el hecho de que han sido expuestas a violencia, o sienten que la violencia que se produce dentro de sus casas es un asunto normal y aceptado, y en otras circunstancias, entienden que el hecho de que exista violencia dentro del hogar, es un asunto de familia y que no debe salir de allí. Tomando en cuenta estos detalles, se debe considerar que los mismos pudiera causar sesgo en la investigación ${ }^{17}$.

\section{Bibliografía}

1. Organización Mundial de la Salud (OMS). (2017) Desarrollo en la adolescencia. Disponible en: https://www.who.int/maternal_child_adolescent/topics/adolescence/ es

2. Villavicencio J, Sandoval W, Arévalo Veras M, Galván M, Lara Caballeros E, López Pérez $S$ et al. Guía de prevención del embarazo en la adolescencia en Guatemala: creciendo juntos responsablemente. Guatemala; (p. 19-25). Disponible en: http://www.paho.org/gut/ index.php?option=com_docman \&view=download\&category_slug=publications\&alias $=618$ - guia-de-prevencion-del-embarazo-en-la-adolescencia-en-guatemala\&Ite$\operatorname{mid}=518$ 
Evaluación de los Factores de Riesgo de Embarazo en Adolescentes en la Comunidad de Las Tablas, Municipio Matanzas, Provincia Peravia, República Dominicana, durante el período noviembre 2017 - enero 2018

3. Bretón $M$, Barinas $S$, Medina S, Díaz J, Fernández Y, Ramírez G. (2017) Programa de las Naciones Unidas para el Desarrollo. Informe Nacional de Desarrollo Humano 2017 - Embarazo Adolescente: Un desafío multidimensional para generar oportunidades en el ciclo de la vida. República Dominicana. Disponible en: http://www.do.undp.org/ content/dominican_republic/es/home/library/human_development/informe-nacional-de-desarrollo-humano-2017---embarazo-adolescent.html

4. UNICEF. (2015) Embarazo en adolescentes. Disponible en: https://www.unicef.org/republicadominicana/health_childhood_25945. htm

5. Organización Mundial de la Salud (OMS). (2017) Adolescentes: riesgos para la salud y soluciones. Disponible en: http://www.who. int/mediacentre/factsheets/fs345/es/

6. Sibec T, Muñiz, M, Maddaleno M, Suárez E. Manual de Medicina de la Adolescencia. Organización Panamericana de la Salud. 2017; p. 473.

7. Alfonso Figueroa L, Figueroa Pérez L. Conductas sexuales de riesgo en adolescentes desde el contexto cubano. Revista de Ciencias Médicas vol.21 no.2. 2017. Disponible en: http://scielo.sld.cu/scielo.php?script=sci_arttext\&pid=S1561-31942017000200020

8. Arana C, Martínez R, Rosales C. (2013) Factores que predisponen los embarazos en las adolescentes. (Tesis de licenciatura). Universidad Nacional del Cuyo. Disponible en: http://bdigital.uncu.edu.ar/objetos_digitales/5987/arana-carina.pdf

9. UNICEF. (2011) Prevención del embarazo en adolescentes. República Dominicana. Disponible en: https:// www.unicef.org/republicadominicana/ prevencion_embarazo_adolescente2011.pdf

10. Organización Mundial de la Salud (OMS). (2014) El embarazo en la adolescencia. Disponible en: http://www.who.int/ mediacentre/factsheets/fs364/es/

11. Pérez Then E, Miric M, Vargas T. Embarazo en adolescentes: ¿¿na realidad en transición? Profamilia. CENISMI. 2011. p. 67.

12. Rodríguez Rodríguez N, Cala Bayeux A, Nápoles Pérez J, Milán Arenado Y, Aguilar Tito M. (2017) Factores de riesgos asociados al embarazo en adolescentes. (Tesis, Universidad de Ciencias Médicas). Disponible en: http:// www.revinfcientifica.sld.cu/index.php/ric/ article/view/27/53

13. Casadiego W. (2014) Conocimiento y uso de métodos anticonceptivos en un grupo de adolescentes (Tesis, Universidad de Carabobo). Valencia, España. Disponible en: http://riuc. bc.uc.edu.ve/bitstream/123456789/549/4/ wcasadiego.pdf

14. UNICEF. (2010) Estudio del Secretario General de la Naciones Unidas sobre la violencia contra los niños. Disponible en: https:// www.unicef.org/lac/hoja1(1).pdf

15. Holguín Y, Mendoza L, Esquivel C, Sánchez R, Daravińa A, et al. (2013) Factores asociados al inicio de la actividad sexual en adolescentes. (Tesis, Universidad Central del Valle). Tuluá, Colombia. Disponible en: http://www.revistasochog.cl/files/pdf/DR.HOLGUIN0.pdf

16. Ávila Montes G, Barbena García M, Arguello Munguía P. (2011) Factores de riesgo asociados al embarazo en adolescentes en comunidades urbano marginales. (Tesis, Universidad Autónoma de Barcelona). Comayagüela. Honduras. Disponible en: http://www.bvs. hn/RMH/pdf/2011/pdf/Vol79-2-2011-5.pdf 
Amelia Navarro Ramírez, Bellelyn Domínguez, Rodríguez Charlotte Montes de Oca, Ana Ramírez Díaz, Nicole Barreto Rojas, César López y Emilton López

17. Guzmán Sánchez F. (2015) Violencia de género en adolescentes: Análisis de las percepciones y de las acciones educativas propuestas por la Junta de Andalucía. (Tesis, Universidad de Sevilla. Sevilla. España. Disponible en: https://idus.us.es/xmlui/bitstream/handle/11441/36522/TESIS\%20COMPLETA. pdf?sequence $=1$ 